\title{
On doubly nonlinear elliptic-parabolic systems arising in coupled transport phenomena in unsaturated porous media
}

\author{
Michal Beneš and Lukáš Krupička
}

\begin{abstract}
We consider an initial-boundary value problem for a fully nonlinear degenerate parabolic system. The mathematical problem is originally motivated by coupled Richards and heat equations modelling hygrothermal flows in unsaturated porous media and fractured rock masses. We prove a global existence of a weak solution to this problem on an arbitrary interval of time.
\end{abstract}

Mathematics Subject Classification (2010). Primary 35A01; Secondary 35K61, 80A20.

Keywords. Initial-boundary value problems for second-order parabolic systems, Global existence of weak solutions, Coupled heat and mass transport in porous media.

\section{Introduction}

Modelling of coupled heat and mass transfer in unsaturated porous media plays a crucial role in many agricultural, environmental and civil engineering applications. The major difficulty in predicting the coupled transport processes in porous media and fractured rock masses lies in their complex porous microstructure, resulting in a highly non-linear dependence of transport coefficients on temperature and water content. Mathematical models of coupled transport processes in porous materials are based on balance equations, governing the conservation of mass and thermal energy, see e.g. [7,24] for a recent survey. Such systems of balance equations can be written in the common general form

$$
\partial_{t} \mathbf{B}(\boldsymbol{u})-\nabla \cdot \mathbf{A}(\boldsymbol{u}, \nabla \boldsymbol{u})=\mathbf{F}(\boldsymbol{u})
$$

Problem of type (1.1) are too complex to be solved analytically. Typically, such equations, supplemented by the appropriate initial and boundary conditions, are solved by discretizing the domain spatially using finite elements or finite 
volumes, then integrating over time using a numerical ordinary differential equation solver. However, to our best knowledge, the qualitative properties of the resulting systems remain largely unexplored.

No general existence, uniqueness and regularity theory for the mentioned problem is developed. Nevertheless, some partial results assuming special structure of operators $\mathbf{A}, \mathbf{B}$ and $\mathbf{F}$ can be found in the literature. The existence, uniqueness and regularity of solutions to the system like (1.1) have been proved by Alt and Luckhaus in [2]. They obtained their results assuming the operator $\mathbf{B}$ to be only monotone and subgradient.

Later on, these results have been disseminated in several directions. Filo and Kačur [10] proved the local existence of the weak solution for the system under nonlinear Neumann boundary conditions and assuming more general growth conditions on nonlinearities in $\boldsymbol{u}$. However, these results are not applicable if $\mathbf{B}$ does not take the subgradient structure, which is the case of coupled balance equations for energy and mass transport. To the best of our knowledge, the only related works in this context are due to Vala [25], Li and Sun [18], Li et al. [19] and Li and Sun [20]. Although the approach in [25] admits non-symmetry in the parabolic term, it requires unrealistic symmetry in the elliptic part. The last-mentioned works, studying a model of specific structure of a heat and mass transfer arising from textile industry, prove the global existence for one-dimensional problems in $[18,19]$ and three-dimensional problems in [20]. Recently, the existence of a local-in-time strong solution for moisture and heat transfer in multi-layer porous structures modelling by the system close to (1.1) is proven in [6].

In this paper we explore the degenerate problem with specific structure of (1.1) resulting from coupled filtration of a fluid with heat energy transfer in partially saturated porous media described by the Richards equation and the convection-diffusion heat equation (see Section 7).

Let $\Omega \in \mathbb{R}^{2}$ be a bounded domain with a smooth boundary $\Gamma$ of class $C^{2}, T \in(0, \infty)$ be fixed throughout the paper. Let us abbreviate $I:=(0, T)$, $Q_{T}:=\Omega \times I$ and $\Gamma_{T}:=\Gamma \times I$. We study the Cauchy-Dirichlet problem

$$
\begin{aligned}
\partial_{t} b(u) & =\nabla \cdot(a(\theta) \nabla u+e(\theta, u)) \quad \text { in } Q_{T}, \\
\partial_{t}(b(u) \theta+\rho \theta) & =\nabla \cdot(\lambda(\theta, u) \nabla \theta)+\nabla \cdot(\theta(a(\theta) \nabla u+e(\theta, u))) \quad \text { in } Q_{T}, \\
u & =0 \quad \text { in } \Gamma_{T}, \\
\theta & =0 \quad \text { in } \Gamma_{T}, \\
u(0) & =u_{0} \quad \text { in } \Omega, \\
\theta(0) & =\theta_{0} \quad \text { in } \Omega .
\end{aligned}
$$

Here $u: Q_{T} \rightarrow \mathbb{R}$ and $\theta: Q_{T} \rightarrow \mathbb{R}$ are the unknowns. $a: \mathbb{R} \rightarrow \mathbb{R}, b: \mathbb{R} \rightarrow \mathbb{R}$, $\lambda: \mathbb{R}^{2} \rightarrow \mathbb{R}, \boldsymbol{e}: \mathbb{R}^{2} \rightarrow \mathbb{R}^{2}, u_{0}: \Omega \rightarrow \mathbb{R}$ and $\theta_{0}: \Omega \rightarrow \mathbb{R}$ are given functions, $\rho$ is a real positive constant and $\boldsymbol{n}$ is an outer normal vector. The aim of this paper is to prove the existence of a weak solution by a carefully designed limit passage in the appropriate regularized problem. To the best of our knowledge, there is no existence result for the presented coupled system available. One of the main novelties of this paper is the analysis of a mollification method applied to the 
coupled degenerate problem (1.2)-(1.7). We notice that the specific structure of the convective term $\nabla \cdot(\theta(a(\theta) \nabla u+e(\theta, u)))$ in the energy equation (1.3) is fundamental to derive a priori estimates on the regularized solutions.

The paper is organized as follows. In Sect. 2, we introduce basic notation and the suitable function spaces and derive some necessary continuous and compact embeddings of Bochner spaces. In Sect. 3, we specify our assumptions on data and coefficient functions in the problem. In Sect. 4, we formulate the problem in the variational sense and state the main result of the paper, the global-in-time existence of the weak solution. The main result is proved by an approximation procedure. This consists in proving the existence of solutions to mollified problems using the Schauder fixed point theorem, for which a priori estimates are obtained. Such procedure is carried out in Sect. 5. Using the limiting procedure we prove the existence of the weak solution to the original problem in Sect. 6. In Sect. 7, we present an application of our theory to selected engineering model of heat and water transport in unsaturated porous media. Finally, let us mention, that some auxiliary results needed in the proof are collected in Appendix A.

\section{Notation, function spaces and some auxiliary results}

Vectors and vector functions are denoted by boldface letters. Throughout the paper, we will always use positive constants $C, c, c_{1}, c_{2}, \ldots$, which are not specified and which may differ from line to line.

We use the following common notation for function spaces. For an arbitrary $p \in[1,+\infty], L^{p}(\Omega)$ and $L^{p}\left(Q_{T}\right)$ denote, respectively, the usual Lebesgue spaces equipped with the norm $\|\cdot\|_{L^{p}(\Omega)}$ and $\|\cdot\|_{L^{p}\left(Q_{T}\right)}$, respectively. $W^{k, p}(\Omega)$, $k \geq 0$ ( $k$ need not to be an integer, see [16]), denotes the usual Sobolev space with the norm $\|\cdot\|_{W^{k, p}(\Omega)}$ and $W_{0}^{k, p}(\Omega)$ is the Sobolev space of functions whose trace on $\Gamma$ vanishes. Let $B$ be an arbitrary Banach space, then $B^{*}$ represents its dual. By $L^{p}(I, B)$ we denote the Bochner space (see [1]).

The following compactness result was established by J.P. Aubin (see [3]) and will be crucial to prove the main proposition of the paper.

Theorem 2.1. Let $B_{0}, B, B_{1}$ be three Banach spaces where $B_{0}, B_{1}$ are reflexive. Suppose that $B_{0}$ is continuously imbedded into $B$, which is also continuously imbedded into $B_{1}$, and imbedding from $B_{0}$ into $B$ is compact. For any given $p_{0}, p_{1}$ with $1<p_{0}, p_{1}<\infty$, let

$$
\mathcal{W}:=\left\{v ; v \in L^{p_{0}}\left(I, B_{0}\right), v_{t} \in L^{p_{1}}\left(I, B_{1}\right)\right\} .
$$

Then the imbedding from $\mathcal{W}$ into $L^{p_{0}}(I, B)$ is compact.

Define the spaces

$$
\begin{array}{ll}
W_{2}^{2,1}\left(Q_{T}\right) \equiv\left\{\phi \in L^{2}\left(I, W^{2,2}(\Omega)\right),\right. & \left.\partial_{t} \phi \in L^{2}\left(Q_{T}\right)\right\}, \\
W_{2}^{1,1}\left(Q_{T}\right) \equiv\left\{\phi \in L^{2}\left(I, W^{1,2}(\Omega)\right),\right. & \left.\partial_{t} \phi \in L^{2}\left(Q_{T}\right)\right\} .
\end{array}
$$


We will often use the following embeddings that are consequences of Theorem 2.1 and interpolation theory:

$$
\begin{aligned}
& W_{2}^{2,1}\left(Q_{T}\right) \hookrightarrow \hookrightarrow L^{1}\left(Q_{T}\right), \\
& W_{2}^{2,1}\left(Q_{T}\right) \hookrightarrow L^{\infty}\left(I, W^{1,2}(\Omega)\right) .
\end{aligned}
$$

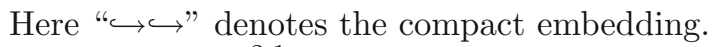

Let $\phi \in W_{2}^{2,1}\left(Q_{T}\right)$. Raising and integrating the interpolation inequality

$$
\|\phi(t)\|_{W^{3 / 2,2}(\Omega)} \leq c\|\phi(t)\|_{W^{1,2}(\Omega)}^{1 / 2}\|\phi(t)\|_{W^{2,2}(\Omega)}^{1 / 2}
$$

from 0 to $T$ we get

$$
\begin{aligned}
\left(\int_{0}^{T}\|\phi(t)\|_{W^{3 / 2,2}(\Omega)}^{4} \mathrm{~d} t\right)^{1 / 4} & \leq c\left(\int_{0}^{T}\|\phi(t)\|_{W^{2,2}(\Omega)}^{2}\|\phi(t)\|_{W^{1,2}(\Omega)}^{2} \mathrm{~d} t\right)^{1 / 4} \\
& \leq c\|\phi\|_{L^{2}\left(I, W^{2,2}(\Omega)\right)}^{1 / 2}\|\phi\|_{L^{\infty}\left(I, W^{1,2}(\Omega)\right)}^{1 / 2} \\
& \leq c\|\phi\|_{W_{2}^{2,1}\left(Q_{T}\right)} .
\end{aligned}
$$

Hence we have

$$
W_{2}^{2,1}\left(Q_{T}\right) \hookrightarrow L^{4}\left(I, W^{3 / 2,2}(\Omega)\right) .
$$

Using the Sobolev embedding

$$
W^{3 / 2,2}(\Omega) \hookrightarrow W^{1,4}(\Omega)
$$

we obtain

$$
W_{2}^{2,1}\left(Q_{T}\right) \hookrightarrow L^{4}\left(I, W^{1,4}(\Omega)\right)
$$

\section{Structure and data properties}

Concerning coefficient functions and data properties, we shall introduce the following hypotheses.

(A1) $b$ is positive and Lipschitz continuous function satisfying

$$
\begin{array}{cll}
0<b(\xi) \leq b_{1}<+\infty & \forall \xi \in \mathbb{R} & \left(b_{1}=\text { const }\right), \\
0<b^{\prime}(\xi) \leq b_{2}<+\infty & \forall \xi \in \mathbb{R} & \left(b_{2}=\text { const }\right) .
\end{array}
$$

(A2) $a$ and $\lambda$ are $C^{1}$-functions, such that

$$
\begin{array}{clrl}
0<a_{1} \leq a(\xi) \leq a_{2}<+\infty & & \forall \xi \in \mathbb{R} \quad\left(a_{1}, a_{2}=\text { const }\right), \\
0<\lambda_{1} \leq \lambda(\xi, \zeta) \leq \lambda_{2}<+\infty & \forall \xi, \zeta \in \mathbb{R} \quad\left(\lambda_{1}, \lambda_{2}=\text { const }\right) .
\end{array}
$$

(A3) $\boldsymbol{e}: \mathbb{R}^{2} \rightarrow \mathbb{R}^{2}$ is continuously differentiable vector-valued function, such that

$$
|\boldsymbol{e}(\xi, \zeta)| \leq e_{1}<+\infty \quad \forall \xi, \zeta \in \mathbb{R} \quad\left(e_{1}=\text { const }\right) .
$$

(A4) For initial data we assume $u_{0}, \theta_{0} \in L^{2}(\Omega)$. 
Remark 3.1. [2, Section 1.1] Let us note that (A1) implies that there is a (strictly) convex $C^{1}$-function $\Phi: \mathbb{R} \rightarrow \mathbb{R}, \Phi(0)=0, \Phi^{\prime}(0)=0$, such that

$$
b(z)-b(0)=\Phi^{\prime}(z) \quad \forall z \in \mathbb{R} .
$$

Introduce the Legendre transform

$$
B(z):=\int_{0}^{1}(b(z)-b(s z)) z \mathrm{~d} s=\int_{0}^{z}(b(z)-b(s)) \mathrm{d} s .
$$

Proposition 3.2 (see [2], Lemma 1.5). Let $u \in L^{2}\left(I, W_{0}^{1,2}(\Omega)\right)$, such that $b(u) \in$ $L^{\infty}\left(I, L^{1}(\Omega)\right), \partial_{t} b(u) \in L^{2}\left(I, W^{1,2}(\Omega)^{*}\right)$, and

$$
\int_{0}^{T}\left\langle\partial_{t} b(u), \phi\right\rangle \mathrm{d} t+\int_{Q_{T}}\left(b(u)-b\left(u_{0}\right)\right) \partial_{t} \phi \mathrm{d} x \mathrm{~d} t=0
$$

for every test function $\phi \in L^{2}\left(I, W_{0}^{1,2}(\Omega)\right) \cap W^{1,1}\left(I, L^{\infty}(\Omega)\right)$ with $\phi(T)=0$. Then

$$
B(u) \in L^{\infty}\left(I, L^{1}(\Omega)\right)
$$

and for almost all $t$ the following formula holds

$$
\int_{\Omega} B(u(t)) \mathrm{d} x-\int_{\Omega} B\left(u_{0}\right) \mathrm{d} x=\int_{0}^{t}\left\langle\partial_{t} b(u), u\right\rangle \mathrm{d} t .
$$

\section{Main result}

We now formulate our problem in a weak sense. The aim of this paper is to prove the existence of a weak solution to the problem described by the system (1.2)-(1.7).

Definition 4.1. We say that a pair $[u, \theta] \in L^{2}\left(I, W_{0}^{1,2}(\Omega)^{2}\right)$ is a weak solution of the system $(1.2)-(1.7)$ iff

$$
-\int_{Q_{T}} b(u) \partial_{t} \phi \mathrm{d} x \mathrm{~d} t+\int_{Q_{T}}(a(\theta) \nabla u+\boldsymbol{e}(\theta, u)) \cdot \nabla \phi \mathrm{d} x \mathrm{~d} t=\int_{\Omega} b\left(u_{0}\right) \phi(0) \mathrm{d} x
$$

and

$$
\begin{aligned}
& -\int_{Q_{T}}(b(u) \theta+\rho \theta) \partial_{t} \psi \mathrm{d} x \mathrm{~d} t+\int_{Q_{T}} \lambda(\theta, u) \nabla \theta \cdot \nabla \psi \mathrm{d} x \mathrm{~d} t \\
& +\int_{Q_{T}}(\theta(a(\theta) \nabla u+e(\theta, u))) \cdot \nabla \psi \mathrm{d} x \mathrm{~d} t \\
& \quad=\int_{\Omega}\left(b\left(u_{0}\right) \theta_{0}+\rho \theta_{0}\right) \psi(0) \mathrm{d} x
\end{aligned}
$$

hold for all test functions $[\phi, \psi] \in C^{\infty}\left(\bar{Q}_{T}\right)^{2}, \phi=\psi=0$ in $\Gamma_{T}$ and $\phi(T)=$ $\psi(T)=0$ in $\Omega$.

The main result of this paper reads as follows.

Theorem 4.2 (Main result). Let the assumptions (A1)-(A4) be satisfied. Then there exists at least one weak solution of the system (1.2)-(1.7). 
In the proof of the main result we use the method of mollification in order to get the approximate solution of the system (1.2)-(1.7). Existence of the strong solution in $W_{2}^{2,1}\left(Q_{T}\right)$ to the regularized system is proved by the Leray-Schauder theorem. Conclusion of the proof consists in establishing a priori estimates on these solutions and then carrying out the passage to the limit.

Remark 4.3. For simplicity, we consider homogeneous boundary conditions. All results in our paper remain valid if one assumes the nonhomogeneous Dirichlet boundary conditions $u=u_{D}$ and $\theta=\theta_{D}$ on $\Gamma_{T}$, where $u_{D}, \theta_{D} \in$ $L^{2}\left(I, W^{1,2}(\Omega)\right) \cap W^{1,1}\left(I, L^{\infty}(\Omega)\right)$.

\section{Solution to the mollified problem}

Let $\theta^{\varepsilon}:=J_{\varepsilon} \star \mathcal{E}(\theta)$ be a regularization of $\theta, \varepsilon$ is a small positive real number, $J_{\varepsilon}$ denotes the standard mollifier in $\mathbb{R}^{3}$ having the support in a ball of radius $\varepsilon$ (see [1, Paragraph 2.28 and Theorem 2.29]) and $\mathcal{E}(\theta)$ is the extension operator extending $\theta$ to be zero on $\mathbb{R}^{3} \backslash Q_{T}$ (see [1, Paragraph 5.17 and Theorem 5.24]). Similarly, we set $u^{\varepsilon}:=J_{\varepsilon} \star \mathcal{E}(u)$.

Let us introduce the mollified system of governing equations

$$
\begin{aligned}
\partial_{t} b(u)= & \nabla \cdot\left(a\left(\theta^{\varepsilon}\right) \nabla u+e\left(\theta^{\varepsilon}, u\right)\right) \quad \text { in } Q_{T}, \\
\partial_{t}(b(u) \theta+\rho \theta)= & \nabla \cdot\left(\lambda\left(\theta^{\varepsilon}, u^{\varepsilon}\right) \nabla \theta\right) \\
& +\nabla \cdot\left(\theta\left(a\left(\theta^{\varepsilon}\right) \nabla u+e\left(\theta^{\varepsilon}, u\right)\right)\right) \quad \text { in } Q_{T} .
\end{aligned}
$$

Substituting (5.1) into (5.2), equations (5.1)-(5.2) can be rewritten as

$$
\begin{aligned}
\partial_{t} b(u)= & \nabla \cdot\left(a\left(\theta^{\varepsilon}\right) \nabla u+e\left(\theta^{\varepsilon}, u\right)\right) \quad \text { in } Q_{T}, \\
(b(u)+\rho) \partial_{t} \theta= & \nabla \cdot\left(\lambda\left(\theta^{\varepsilon}, u^{\varepsilon}\right) \nabla \theta\right) \\
& +\left(a\left(\theta^{\varepsilon}\right) \nabla u+e\left(\theta^{\varepsilon}, u\right)\right) \cdot \nabla \theta \quad \text { in } Q_{T} .
\end{aligned}
$$

The governing equations (5.3)-(5.4) are appended by the following homogeneous boundary conditions and regularized compatible initial conditions

$$
\begin{array}{rc}
u=0 \quad \text { in } \Gamma_{T}, \\
\theta=0 \quad \text { in } \Gamma_{T}, \\
u(0)=u_{0}^{\varepsilon} \quad \text { in } \Omega, \\
\theta(0)=\theta_{0}^{\varepsilon} \quad \text { in } \Omega .
\end{array}
$$

Here and in what follows, we set $\theta_{0}^{\varepsilon}:=J_{\varepsilon} \star\left(\omega_{\varepsilon} \mathcal{E}\left(\theta_{0}\right)\right)$, where

$$
\omega_{\varepsilon}(x):= \begin{cases}0 & \text { if } \operatorname{dist}(x, \partial \Omega) \leq 2 \varepsilon, \\ 1 & \text { elsewhere. }\end{cases}
$$

Similarly, we introduce $u_{0}^{\varepsilon}:=J_{\varepsilon} \star\left(\omega_{\varepsilon} \mathcal{E}\left(u_{0}\right)\right)$. Note that $\theta_{0}^{\varepsilon}, u_{0}^{\varepsilon} \in C_{0}^{\infty}(\Omega)$ (cf. [1, Theorem $2.29(\mathrm{~b})])$ and $\left\|\theta_{0}^{\varepsilon}-\theta_{0}\right\|_{L^{2}(\Omega)} \rightarrow 0$ and $\left\|u_{0}^{\varepsilon}-u_{0}\right\|_{L^{2}(\Omega)} \rightarrow 0$ as $\varepsilon \rightarrow 0_{+}$.

In this section we prove the existence of a strong solution to the regularized problem (5.3)-(5.8).

Theorem 5.1. The problem (5.3)-(5.8) has a strong solution $[u, \theta] \in W_{2}^{2,1}\left(Q_{T}\right)^{2}$. 
Proof of Theorem 5.1 follows from a priori estimates by the Schauder fixed point arguments. In particular, the proof is split into several parts. First, we prove the existence of the strong solution to an auxiliary problem, i.e. for some given $\tilde{\theta} \in L^{1}\left(Q_{T}\right)$ to find $[u, \theta]$, the strong solution to the following system (5.9)-(5.14). Using the embedding (2.1) we get compactness. After that we prove some a priori estimates to get the existence of the fixed point denoted by $\left[u_{\varepsilon}, \theta_{\varepsilon}\right]$ via the following well-known theorem.

Theorem 5.2 (Schauder fixed point theorem). Let $\mathfrak{S}$ be a closed convex set in a Banach space $\mathfrak{B}$ and let $\mathcal{T}$ be a continuous mapping of $\mathfrak{S}$ into itself such that the image $\mathcal{T} \mathfrak{S}$ is precompact. Then $\mathcal{T}$ has a fixed point.

For the proof of Theorem 5.2 see [13, p. 279, Theorem 11.1 and p. 280, Corollary 11.2].

Proof of Theorem 5.1 For any given $\tilde{\theta} \in L^{1}\left(Q_{T}\right)$ consider the initial-boundary value problem

$$
\begin{aligned}
\partial_{t} b(u)= & \nabla \cdot\left(a\left(\tilde{\theta}^{\varepsilon}\right) \nabla u+\boldsymbol{e}\left(\tilde{\theta}^{\varepsilon}, u\right)\right) \quad \text { in } Q_{T}, \\
(b(u)+\rho) \partial_{t} \theta= & \nabla \cdot\left(\lambda\left(\tilde{\theta}^{\varepsilon}, u^{\varepsilon}\right) \nabla \theta\right) \\
& +\left(a\left(\tilde{\theta}^{\varepsilon}\right) \nabla u+\boldsymbol{e}\left(\tilde{\theta}^{\varepsilon}, u\right)\right) \cdot \nabla \theta \quad \text { in } Q_{T}, \\
u= & 0 \quad \text { in } \Gamma_{T}, \\
\theta= & 0 \quad \text { in } \Gamma_{T}, \\
u(0)= & u_{0}^{\varepsilon} \quad \text { in } \Omega, \\
\theta(0)= & \theta_{0}^{\varepsilon} \quad \text { in } \Omega .
\end{aligned}
$$

Step 1. Here we treat the decoupled problem

$$
\begin{aligned}
\partial_{t} b(u) & =\nabla \cdot\left(a\left(\tilde{\theta}^{\varepsilon}\right) \nabla u+\boldsymbol{e}\left(\tilde{\theta}^{\varepsilon}, u\right)\right) \quad \text { in } Q_{T}, \\
u & =0 \quad \text { in } \Gamma_{T}, \\
u(0) & =u_{0}^{\varepsilon} \quad \text { in } \Omega,
\end{aligned}
$$

for which there exists the unique strong solution $u \in W_{2}^{2,1}\left(Q_{T}\right) \cap L^{\infty}\left(Q_{T}\right)$, such that

$$
\left\|\partial_{t} u\right\|_{L^{2}\left(Q_{T}\right)}+\|u\|_{L^{2}\left(I, W_{0}^{1,2}(\Omega)\right)}+\|u\|_{L^{\infty}\left(Q_{T}\right)} \leq C_{u_{0}^{\varepsilon}}
$$

Here the constant $C_{u_{0}^{\varepsilon}}$ is independent of $\tilde{\theta}^{\varepsilon}$. For reader's convenience we do not interrupt this step by the proof of the existence of the unique solution $u \in W_{2}^{2,1}\left(Q_{T}\right)$ to the system (5.15)-(5.17) satisfying (5.18). Therefore, the detailed proof is postponed to Appendix A. 
Step 2. Now having $u \in W_{2}^{2,1}\left(Q_{T}\right)$ we get $\theta$ as the solution of the problem (5.10), (5.12) and (5.14). We can write this linear parabolic problem in the form

$$
\begin{aligned}
\sigma(x, t) \partial_{t} \theta-\nabla \cdot(a(x, t) \nabla \theta)-\boldsymbol{b}(x, t) \cdot \nabla \theta & =0 \quad \text { in } Q_{T}, \\
\theta & =0 \quad \text { in } \Gamma_{T}, \\
\theta(0) & =\theta_{0}^{\varepsilon} \quad \text { in } \Omega,
\end{aligned}
$$

where

$$
\begin{aligned}
\sigma & =b(u)+\rho, \\
a & =\lambda\left(\tilde{\theta}^{\varepsilon}, u^{\varepsilon}\right), \\
\boldsymbol{b} & =a\left(\tilde{\theta}^{\varepsilon}\right) \nabla u+\boldsymbol{e}\left(\tilde{\theta}^{\varepsilon}, u\right) .
\end{aligned}
$$

Here $\sigma \in L^{\infty}\left(Q_{T}\right)$ and $a \in C^{1}\left(Q_{T}\right)$. Since $u \in W_{2}^{2,1}\left(Q_{T}\right) \hookrightarrow L^{4}\left(I, W^{1,4}(\Omega)\right)$ we have $\boldsymbol{b} \in L^{4}\left(I, L^{4}(\Omega)^{2}\right)$, recall the embedding (2.5). By the linear theory for parabolic problems with nonsmooth coefficients (see [17, Chapter III.]) the problem (5.19)-(5.21) has a unique weak solution $\theta \in W_{2}^{1,1}\left(Q_{T}\right)$. Let us further regularize $\theta$. We now write the system (5.19)-(5.21) in the form

$$
\begin{aligned}
\partial_{t} \theta-\nabla \cdot(a(x, t) \nabla \theta)-\boldsymbol{b}(x, t) \cdot \nabla \theta & =f(x, t) \quad \text { in } Q_{T}, \\
\theta & =0 \quad \text { in } \Gamma_{T}, \\
\theta(0) & =\theta_{0}^{\varepsilon} \quad \text { in } \Omega,
\end{aligned}
$$

where

$$
f:=\partial_{t} \theta-\sigma(x, t) \partial_{t} \theta
$$

From the previous steps we have $f \in L^{2}\left(Q_{T}\right)$. Following the linear theory for parabolic equations [17, Chapter III., Remark 6.3.] we get the uniquely determined strong solution of the system (5.19)-(5.21)

$$
\theta \in W_{2}^{2,1}\left(Q_{T}\right)
$$

Step 3. For any given $\tilde{\theta} \in L^{1}\left(Q_{T}\right)$ we have $\theta \in W_{2}^{2,1}\left(Q_{T}\right)$ as the unique solution of the problem (5.10), (5.12) and (5.14), where $u$ solves uniquely the problem (5.9), (5.11) and (5.13). Define the nonlinear mapping $\mathcal{A}: L^{1}\left(Q_{T}\right) \rightarrow L^{1}\left(Q_{T}\right)$, given by the equation

$$
\theta=\mathcal{A}(\tilde{\theta})
$$

Step 4. Here we prove some uniform estimates for all $\theta$ satisfying the equation $\theta=\mathcal{A}(\tilde{\theta})$. Test (5.9) by $\theta^{2}$ and (5.10) by $2 \theta$ to obtain, adding these both resulting equations,

$$
\int_{Q_{t}} \frac{\partial}{\partial s}\left[\theta^{2}(b(u)+\rho)\right] \mathrm{d} x \mathrm{~d} s+\int_{Q_{t}} 2 \lambda\left(\tilde{\theta}^{\varepsilon}, u^{\varepsilon}\right)|\nabla \theta|^{2} \mathrm{~d} x \mathrm{~d} s=0 .
$$

Now, using (3.4) we deduce

$$
\int_{\Omega} \theta^{2}(b(u)+\rho) \mathrm{d} x+2 \lambda_{1} \int_{Q_{t}}|\nabla \theta|^{2} \mathrm{~d} x \mathrm{~d} s \leq \int_{\Omega} \theta_{0}^{2}\left(b\left(u_{0}\right)+\rho\right) \mathrm{d} x .
$$


Hence

$$
\int_{\Omega} \theta(t)^{2} \mathrm{~d} x+\int_{0}^{t}\|\theta(s)\|_{W^{1,2}(\Omega)}^{2} \mathrm{~d} s \leq c,
$$

which immediately yields

$$
\|\theta\|_{L^{\infty}\left(I, L^{2}(\Omega)\right)}+\|\theta\|_{L^{2}\left(I, W^{1,2}(\Omega)\right)} \leq c
$$

for some positive constant $c$ (independent of $\tilde{\theta}$ ).

Step 5. Now we prove the continuity of the mapping $\mathcal{A}$. For $\tilde{\theta} \in L^{1}\left(Q_{T}\right)$ we solve $(5.15)-(5.17)$. Setting $u=\mathcal{A}_{1}(\tilde{\theta})$, to denote the solution of this problem, we solve

$$
\begin{aligned}
&(b(u)+\rho) \partial_{t} \theta=\nabla \cdot\left(\lambda\left(\tilde{\theta}^{\varepsilon}, u^{\varepsilon}\right) \nabla \theta\right) \\
&+\left(a\left(\tilde{\theta}^{\varepsilon}\right) \nabla u+e\left(\tilde{\theta}^{\varepsilon}, u\right)\right) \cdot \nabla \theta \quad \text { in } Q_{T}, \\
& \theta=0 \quad \text { in } \Gamma_{T} \\
& \theta(0)=\theta_{0}^{\varepsilon} \quad \text { in } \Omega .
\end{aligned}
$$

Denote by $\theta=\mathcal{A}_{2}(u, \tilde{\theta})$ the solution of this problem and define $\mathcal{A}(\tilde{\theta})=\mathcal{A}_{3}(\theta)$, where $\mathcal{A}_{3}$ is the embedding of $W_{2}^{2,1}\left(Q_{T}\right)$ into $L^{1}\left(Q_{T}\right)$.

Let $\tilde{\theta}_{n} \in L^{1}\left(Q_{T}\right)$ be a sequence converging strongly to $\tilde{\theta}$ in $L^{1}\left(Q_{T}\right)$. Following Step 1 there exists a sequence of $u_{n}=\mathcal{A}_{1}\left(\tilde{\theta}_{n}\right) \in W_{2}^{2,1}\left(Q_{T}\right) \cap L^{\infty}\left(Q_{T}\right)$, such that

$$
\int_{Q_{T}} \partial_{t} b\left(u_{n}\right) \phi \mathrm{d} x \mathrm{~d} t+\int_{Q_{T}}\left(a\left(\tilde{\theta}_{n}^{\varepsilon}\right) \nabla u_{n}+\boldsymbol{e}\left(\tilde{\theta}_{n}^{\varepsilon}, u_{n}\right)\right) \cdot \nabla \phi \mathrm{d} x \mathrm{~d} t=0
$$

for any test function $\phi \in W_{2}^{1,2}\left(Q_{T}\right), \phi=0$ in $\Gamma_{T}$.

It follows from the estimate (5.18) that there exists a function $u \in$ $W_{2}^{1,1}\left(Q_{T}\right)$, such that (along a selected subsequence) we have

$$
u_{n} \rightarrow u \text { weakly in } W_{2}^{1,1}\left(Q_{T}\right) \text {. }
$$

By the compact embedding $W_{2}^{1,1}\left(Q_{T}\right) \hookrightarrow \hookrightarrow L^{1}\left(Q_{T}\right)$ we have

$$
u_{n} \rightarrow u \text { strongly in } L^{1}\left(Q_{T}\right)
$$

and therefore

$$
u_{n} \rightarrow u \text { almost everywhere in } Q_{T} .
$$

From (5.33) we deduce

$$
\begin{aligned}
& \int_{Q_{T}} \partial_{t} b\left(u_{n}\right) \phi \mathrm{d} x \mathrm{~d} t+\int_{Q_{T}} a\left(\tilde{\theta}^{\varepsilon}\right) \nabla u_{n} \cdot \nabla \phi \mathrm{d} x \mathrm{~d} t \\
& +\int_{Q_{T}}\left[a\left(\tilde{\theta}_{n}^{\varepsilon}\right)-a\left(\tilde{\theta}^{\varepsilon}\right)\right] \nabla u_{n} \cdot \nabla \phi \mathrm{d} x \mathrm{~d} t+\int_{Q_{T}} \boldsymbol{e}\left(\tilde{\theta}^{\varepsilon}, u_{n}\right) \cdot \nabla \phi \mathrm{d} x \mathrm{~d} t \\
& \quad+\int_{Q_{T}}\left(\boldsymbol{e}\left(\tilde{\theta}_{n}^{\varepsilon}, u_{n}\right)-\boldsymbol{e}\left(\tilde{\theta}^{\varepsilon}, u_{n}\right)\right) \cdot \nabla \phi \mathrm{d} x \mathrm{~d} t=0
\end{aligned}
$$


for any test function $\phi \in W_{2}^{1,2}\left(Q_{T}\right), \phi=0$ in $\Gamma_{T}$. We now proceed using ideas in $[14,22]$. By the Lebesgue dominated convergence theorem we conclude (along a selected subsequence)

$$
\begin{aligned}
& \int_{Q_{T}}\left[a\left(\tilde{\theta}_{n}^{\varepsilon}\right)-a\left(\tilde{\theta}^{\varepsilon}\right)\right] \nabla u_{n} \cdot \nabla \phi \mathrm{d} x \mathrm{~d} t \rightarrow 0, \\
& \int_{Q_{T}}\left(\boldsymbol{e}\left(\tilde{\theta}_{n}^{\varepsilon}, u_{n}\right)-\boldsymbol{e}\left(\tilde{\theta}^{\varepsilon}, u_{n}\right)\right) \cdot \nabla \phi \mathrm{d} x \mathrm{~d} t \rightarrow 0
\end{aligned}
$$

and by (5.34) and (5.36) we arrive at the following limits (along a selected subsequence)

$$
\begin{aligned}
& \int_{Q_{T}} \partial_{t} b\left(u_{n}\right) \phi \mathrm{d} x \mathrm{~d} t \rightarrow \int_{Q_{T}} \partial_{t} b(u) \phi \mathrm{d} x \mathrm{~d} t, \\
& \int_{Q_{T}} a\left(\tilde{\theta}^{\varepsilon}\right) \nabla u_{n} \cdot \nabla \phi \mathrm{d} x \mathrm{~d} t \rightarrow \int_{Q_{T}} a\left(\tilde{\theta}^{\varepsilon}\right) \nabla u \cdot \nabla \phi \mathrm{d} x \mathrm{~d} t, \\
& \int_{Q_{T}} e\left(\tilde{\theta}^{\varepsilon}, u_{n}\right) \cdot \nabla \phi \mathrm{d} x \mathrm{~d} t \rightarrow \int_{Q_{T}} e\left(\tilde{\theta}^{\varepsilon}, u\right) \cdot \nabla \phi \mathrm{d} x \mathrm{~d} t .
\end{aligned}
$$

Consequently, as $\tilde{\theta}_{n} \rightarrow \tilde{\theta}$ in $L^{1}\left(Q_{T}\right)$ we have (along a selected subsequence) $\mathcal{A}_{1}\left(\tilde{\theta}_{n}\right) \rightarrow \mathcal{A}_{1}(\tilde{\theta})$ weakly in $W_{2}^{1,1}\left(Q_{T}\right)$.

By the uniqueness of the solution it follows, that the convergence holds for the whole sequence.

Following Step 2 there exists a sequence of $\theta_{n}=\mathcal{A}_{2}\left(u_{n}, \tilde{\theta}_{n}\right) \in W_{2}^{2,1}\left(Q_{T}\right)$, such that the variational equation

$$
\begin{aligned}
& \int_{0}^{T}\left\langle\partial_{t}\left(b\left(u_{n}\right) \theta_{n}+\rho \theta_{n}\right), \psi\right\rangle \mathrm{d} t+\int_{Q_{T}} \lambda\left(\tilde{\theta}_{n}^{\varepsilon}, u_{n}^{\varepsilon}\right) \nabla \theta_{n} \cdot \nabla \psi \mathrm{d} x \mathrm{~d} t \\
& \quad+\int_{Q_{T}} \theta_{n}\left(a\left(\tilde{\theta}_{n}^{\varepsilon}\right) \nabla u_{n}+\boldsymbol{e}\left(\tilde{\theta}_{n}^{\varepsilon}, u_{n}\right)\right) \cdot \nabla \psi \mathrm{d} x \mathrm{~d} t=0
\end{aligned}
$$

holds for all $\psi \in C^{\infty}\left(\bar{Q}_{T}\right), \psi=0$ in $\Gamma_{T}$. In what follows, we show that $\theta_{n}=\mathcal{A}_{2}\left(u_{n}, \tilde{\theta}_{n}\right) \rightarrow \theta=\mathcal{A}_{2}(u, \tilde{\theta})$ strongly in $L^{1}\left(Q_{T}\right)$ and $\theta$ satisfies (5.37). It follows then that the operator $\mathcal{A}$ is continuous.

Raising and integrating the interpolation inequality (cf. [1, Theorem 5.2])

$$
\|\phi(t)\|_{L^{10 / 3}(\Omega)} \leq c\|\phi(t)\|_{W^{1,2}(\Omega)}^{3 / 5}\|\phi(t)\|_{L^{2}(\Omega)}^{2 / 5}
$$

from 0 to $T$ we get

$$
\begin{aligned}
\left(\int_{0}^{T}\|\phi(t)\|_{L^{10 / 3}(\Omega)}^{10 / 3} \mathrm{~d} t\right)^{3 / 10} & \leq c\left(\int_{0}^{T}\|\phi(t)\|_{W^{1,2}(\Omega)}^{2}\|\phi(t)\|_{L^{2}(\Omega)}^{4 / 3} \mathrm{~d} t\right)^{3 / 10} \\
& \leq c\|\phi\|_{L^{2}\left(I, W^{1,2}(\Omega)\right)}^{3 / 5}\|\phi\|_{L^{\infty}\left(I, L^{2}(\Omega)\right)}^{2 / 5}
\end{aligned}
$$

Hence we have

$$
L^{2}\left(I, W^{1,2}(\Omega)\right) \cap L^{\infty}\left(I, L^{2}(\Omega)\right) \hookrightarrow L^{10 / 3}\left(Q_{T}\right) .
$$


Now using (5.18), (5.29), (5.38) and (5.39) we get the uniform bound

$$
\begin{aligned}
& \left\|\theta_{n}\left[a\left(\theta_{n}^{\varepsilon}\right) \nabla u_{n}+\boldsymbol{e}\left(\theta_{n}^{\varepsilon}, u_{n}\right)\right]\right\|_{L^{5 / 4}\left(Q_{T}\right)^{2}} \\
& \quad \leq\left\|\theta_{n}\right\|_{L^{10 / 3}\left(Q_{T}\right)}\left(\left\|a\left(\theta_{n}^{\varepsilon}\right) \nabla u_{n}\right\|_{L^{2}\left(Q_{T}\right)^{2}}+\left\|\boldsymbol{e}\left(\theta_{n}^{\varepsilon}, u_{n}\right)\right\|_{L^{2}\left(Q_{T}\right)^{2}}\right) \leq c
\end{aligned}
$$

and similarly

$$
\left\|b\left(u_{n}\right) \theta_{n}+\rho \theta_{n}\right\|_{L^{5 / 4}\left(I, W^{1,5 / 4}(\Omega)\right)} \leq c .
$$

From the equation (5.2) we obtain the uniform estimate

$$
\left\|\partial_{t}\left(b\left(u_{n}\right) \theta_{n}+\rho \theta_{n}\right)\right\|_{L^{5 / 4}\left(I, W^{1,5}(\Omega)^{*}\right)} \leq c .
$$

Since

$$
W^{1,5 / 4}(\Omega) \hookrightarrow \hookrightarrow W^{1-\beta, 5 / 4}(\Omega) \hookrightarrow W^{1,5}(\Omega)^{*},
$$

where $\beta$ is a small positive real number, the Aubin-Lions lemma yields the existence of $\chi \in L^{5 / 4}\left(I, W^{1-\beta, 5 / 4}(\Omega)\right)$ such that (modulo a subsequence)

$$
b\left(u_{n}\right) \theta_{n}+\rho \theta_{n} \rightarrow \chi \quad \text { strongly in } L^{5 / 4}\left(I, W^{1-\beta, 5 / 4}(\Omega)\right) .
$$

Since (6.17) yields the almost everywhere convergence and $u_{n}$ converges almost everywhere to $u$, we conclude

$$
\theta_{n} \rightarrow \theta \quad \text { almost everywhere in } Q_{T} .
$$

Hence, $b\left(u_{n}\right) \theta_{n}+\rho \theta_{n}$ converges almost everywhere to $b(u) \theta+\rho \theta$ and

$$
\chi=b(u) \theta+\rho \theta .
$$

The above established convergences are sufficient for taking the limit $n \rightarrow \infty$ to get (along a selected subsequence)

$$
\begin{gathered}
\int_{0}^{T}\left\langle\partial_{t}\left(b\left(u_{n}\right) \theta_{n}+\rho \theta_{n}\right), \psi\right\rangle \mathrm{d} t \rightarrow \int_{0}^{T}\left\langle\partial_{t}(b(u) \theta+\rho \theta), \psi\right\rangle \mathrm{d} t \\
\int_{Q_{T}} \lambda\left(\tilde{\theta}_{n}^{\varepsilon}, u_{n}\right) \nabla \theta_{n} \cdot \nabla \psi \mathrm{d} x \mathrm{~d} t \rightarrow \int_{Q_{T}} \lambda\left(\tilde{\theta}^{\varepsilon}, u\right) \nabla \theta \cdot \nabla \psi \mathrm{d} x \mathrm{~d} t \\
\int_{Q_{T}} \theta_{n}\left(a\left(\tilde{\theta}_{n}^{\varepsilon}\right) \nabla u_{n}+\boldsymbol{e}\left(\tilde{\theta}_{n}^{\varepsilon}, u_{n}\right)\right) \cdot \nabla \psi \mathrm{d} x \mathrm{~d} t \\
\rightarrow \int_{Q_{T}} \theta\left(a\left(\tilde{\theta}^{\varepsilon}\right) \nabla u+\boldsymbol{e}\left(\tilde{\theta}^{\varepsilon}, u\right)\right) \cdot \nabla \psi \mathrm{d} x \mathrm{~d} t .
\end{gathered}
$$

Uniqueness of the solution implies that the above convergences hold for the whole sequence.

Finally, simple calculation yields

$$
\theta-\theta_{n}=\frac{\left(b\left(u_{n}\right)+\rho\right) \theta_{n}-(b(u)+\rho) \theta}{b(u)+\rho}-\frac{\left(b\left(u_{n}\right)-b(u)\right) \theta_{n}}{b(u)+\rho} .
$$

By (5.35), (5.43) and (5.45) the right hand side tends to zero in $L^{1}\left(Q_{T}\right)$, therefore

$$
\left\|\theta-\theta_{n}\right\|_{L^{1}\left(Q_{T}\right)} \rightarrow 0 .
$$

Now we conclude, that the operator $\mathcal{A}$ is continuous and, by compactness of $\mathcal{A}_{3}$, completely continuous. 
Step 6 . We note that the operator $\mathcal{A}$ is completely continuous and, by the a priori estimate (5.29) and continuous embedding $L^{2}\left(I, W^{1,2}(\Omega)\right) \hookrightarrow L^{1}\left(Q_{T}\right)$,

$\theta$ is contained in some fixed ball of $L^{1}\left(Q_{T}\right)$ with radius independent of $\tilde{\theta}$. Now we conclude the proof of Theorem 5.1 applying Theorem 5.2.

\section{Proof of the main result}

Let us denote by $\left[u_{\varepsilon}, \theta_{\varepsilon}\right] \in W_{2}^{2,1}\left(Q_{T}\right)^{2}$ the solution to the system (5.3)-(5.8) ensured by Theorem 5.1 .

\subsection{Uniform estimates with respect to $\varepsilon$}

Here we prove some uniform estimates (with respect to $\varepsilon$ ) for the couple $\left[u_{\varepsilon}, \theta_{\varepsilon}\right] \in W_{2}^{2,1}\left(Q_{T}\right)^{2}$, the solution to the system (5.3)-(5.8). In the sequel, $C$ denotes the generic constant being independent of $\varepsilon$, which can vary from line to line.

Multiplying the equation (5.3) by $u_{\varepsilon}$ and integrating over $Q_{t}$ we get

$$
\begin{aligned}
& \int_{\Omega} B\left(u_{\varepsilon}(t)\right) \mathrm{d} x+\int_{Q_{t}} a\left(\theta_{\varepsilon}^{\varepsilon}\right)\left|\nabla u_{\varepsilon}\right|^{2} \mathrm{~d} x \mathrm{~d} s \\
& =\int_{\Omega} B\left(u_{\varepsilon}(0)\right) \mathrm{d} x+\int_{Q_{t}} e\left(\theta_{\varepsilon}^{\varepsilon}, u_{\varepsilon}\right) \cdot \nabla u_{\varepsilon} \mathrm{d} x \mathrm{~d} s
\end{aligned}
$$

and applying the Young's inequality to the last integral we arrive at the estimate

$$
\int_{0}^{t}\left\|u_{\varepsilon}(s)\right\|_{W^{1,2}(\Omega)}^{2} \mathrm{~d} s \leq C .
$$

Hence, we get

$$
\left\|u_{\varepsilon}\right\|_{L^{2}\left(I, W^{1,2}(\Omega)\right)} \leq C
$$

Now let us derive some uniform estimates for $\theta_{\varepsilon}$. The same way as in (5.26)-(5.29), test (5.3) by $\theta_{\varepsilon}^{2}$ and (5.4) by $2 \theta_{\varepsilon}$ to obtain

$$
\int_{Q_{t}} \frac{\partial}{\partial s}\left[\theta_{\varepsilon}^{2}\left(b\left(u_{\varepsilon}\right)+\rho\right)\right] \mathrm{d} x \mathrm{~d} s+\int_{Q_{t}} 2 \lambda\left(\theta_{\varepsilon}^{\varepsilon}, u_{\varepsilon}^{\varepsilon}\right)\left|\nabla \theta_{\varepsilon}\right|^{2} \mathrm{~d} x \mathrm{~d} s=0 .
$$

Applying (3.4) we arrive at the inequality

$$
\int_{\Omega} \theta_{\varepsilon}^{2}\left(b\left(u_{\varepsilon}\right)+\rho\right) \mathrm{d} x+2 \lambda_{1} \int_{Q_{t}}\left|\nabla \theta_{\varepsilon}\right|^{2} \mathrm{~d} x \mathrm{~d} s \leq \int_{\Omega} \theta_{\varepsilon}^{2}(0)\left(b\left(u_{\varepsilon}(0)\right)+\rho\right) \mathrm{d} x .
$$

Therefore

$$
\left\|\theta_{\varepsilon}(t)\right\|_{L^{2}(\Omega)}^{2}+\int_{0}^{t}\left\|\theta_{\varepsilon}(s)\right\|_{W^{1,2}(\Omega)}^{2} \mathrm{~d} s \leq C,
$$

which immediately yields

$$
\begin{aligned}
\left\|\theta_{\varepsilon}\right\|_{L^{2}\left(I, W^{1,2}(\Omega)\right)} & \leq C, \\
\left\|\theta_{\varepsilon}\right\|_{L^{\infty}\left(I, L^{2}(\Omega)\right)} & \leq C .
\end{aligned}
$$

Having established the a priori estimates we are ready to complete the proof of the main result passing to the limit for $\varepsilon \rightarrow 0$. 


\subsection{Passage to the limit}

Here we pass to the limit for $\varepsilon \rightarrow 0$ and study the convergence of the solution

$$
\left[u_{\varepsilon}, \theta_{\varepsilon}\right] \in W_{2}^{2,2}\left(Q_{T}\right)^{2}
$$

of the system (5.3)-(5.8). We present various convergence results based on the uniform estimates (6.3) and (6.7)-(6.8) and, in addition, mention some a priori estimates for the strong solution $\left[u_{\varepsilon}, \theta_{\varepsilon}\right]$ of the system $(5.3)-(5.4)$, which is equivalent to the problem (5.1)-(5.2), with the boundary and initial conditions (5.5)-(5.8).

First, recall the a priori estimates

$$
\begin{gathered}
\left\|u_{\varepsilon}\right\|_{L^{2}\left(I, W^{1,2}(\Omega)\right)} \leq C \\
\left\|\theta_{\varepsilon}\right\|_{L^{2}\left(I, W^{1,2}(\Omega)\right)},\left\|\theta_{\varepsilon}\right\|_{L^{\infty}\left(I, L^{2}(\Omega)\right)} \leq C .
\end{gathered}
$$

As a consequence of the preceding a priori estimates we see that there exist functions $u \in L^{2}\left(I, W^{1,2}(\Omega)\right)$ and $\theta \in L^{2}\left(I, W^{1,2}(\Omega)\right)$, such that, along a selected subsequence, we have $\left(\varepsilon_{j} \rightarrow 0^{+}\right.$as $\left.j \rightarrow \infty\right)$

$$
\begin{aligned}
& u_{\varepsilon_{j}} \rightarrow u \quad \text { weakly in } L^{2}\left(I, W^{1,2}(\Omega)\right), \\
& \theta_{\varepsilon_{j}} \rightarrow \theta \quad \text { weakly in } L^{2}\left(I, W^{1,2}(\Omega)\right) .
\end{aligned}
$$

Using the compactness argument one can show in the same way as in $[2$, Lemma 1.9] and [10, Eqs. (2.10)-(2.12)]

$$
b\left(u_{\varepsilon_{j}}\right) \rightarrow b(u) \text { in } L^{1}\left(Q_{T}\right)
$$

and almost everywhere in $Q_{T}$.

Since $b$ is strictly monotone, it follows from (6.13) that $[15$, Proposition $3.35]$

$$
u_{\varepsilon_{j}} \rightarrow u \quad \text { almost everywhere in } Q_{T} .
$$

We now proceed the same way as in (5.41)-(5.45). Using (6.9), (6.10), (5.38) and (5.39) we get the following bounds

$$
\begin{aligned}
& \left\|b\left(u_{\varepsilon}\right) \theta_{\varepsilon}+\rho \theta_{\varepsilon}\right\|_{L^{5 / 4}\left(I_{\left.1, W^{1,5 / 4}(\Omega)\right)} \leq C\right.}, \\
& \left\|\partial_{t}\left(b\left(u_{\varepsilon}\right) \theta_{\varepsilon}+\rho \theta_{\varepsilon}\right)\right\|_{L^{5 / 4}\left(I, W^{1,5}(\Omega)^{*}\right)} \leq C .
\end{aligned}
$$

Using Aubin-Lions lemma we conclude that there exists

$$
\chi \in L^{5 / 4}\left(I, W^{1-\beta, 5 / 4}(\Omega)\right),
$$

where $\beta$ is a small positive number, such that (along a selected subsequence)

$$
b\left(u_{\varepsilon_{j}}\right) \theta_{\varepsilon_{j}}+\rho \theta_{\varepsilon_{j}} \rightarrow \chi \quad \text { strongly in } L^{5 / 4}\left(I, W^{1-\beta, 5 / 4}(\Omega)\right) .
$$

Since $u_{\varepsilon_{j}}$ converges almost everywhere to $u$, we conclude using (6.17)

$$
\theta_{\varepsilon_{j}} \rightarrow \theta \quad \text { almost everywhere in } Q_{T} .
$$

Hence, $b\left(u_{\varepsilon_{j}}\right) \theta_{\varepsilon_{j}}+\rho \theta_{\varepsilon_{j}}$ converges almost everywhere to $b(u) \theta+\rho \theta$ and $\chi=$ $b(u) \theta+\rho \theta$. Finally, (6.16) yields

$$
\partial_{t}\left(b\left(u_{\varepsilon_{j}}\right) \theta_{\varepsilon_{j}}+\rho \theta_{\varepsilon_{j}}\right) \rightarrow \partial_{t}(b(u) \theta+\rho \theta) \text { weakly in } L^{5 / 4}\left(I, W^{1,5}(\Omega)^{*}\right) .
$$


By (6.11), (6.12) and (6.18) we conclude

$$
\begin{gathered}
\lambda\left(\theta_{\varepsilon_{j}}^{\varepsilon}, u_{\varepsilon_{j}}^{\varepsilon}\right) \nabla \theta_{\varepsilon_{j}} \rightarrow \lambda(\theta, u) \nabla \theta \quad \text { weakly in } L^{2}\left(Q_{T}\right)^{2}, \\
a\left(\theta_{\varepsilon_{j}}^{\varepsilon}\right) \nabla u_{\varepsilon_{j}}+e\left(\theta_{\varepsilon_{j}}^{\varepsilon}, u_{\varepsilon_{j}}\right) \rightarrow a(\theta) \nabla u+e(\theta, u) \text { weakly in } L^{2}\left(Q_{T}\right)^{2}
\end{gathered}
$$

and finally,

$\theta_{\varepsilon_{j}}\left(a\left(\theta_{\varepsilon_{j}}^{\varepsilon}\right) \nabla u_{\varepsilon_{j}}+e\left(\theta_{\varepsilon_{j}}^{\varepsilon}, u_{\varepsilon_{j}}\right)\right) \rightarrow \theta(a(\theta) \nabla u+e(\theta, u))$ weakly in $L^{5 / 4}\left(Q_{T}\right)^{2}$.

The strong solution $\left[u_{\varepsilon}, \theta_{\varepsilon}\right]$ of the problem (5.3)-(5.8) (ensured by Theorem 5.1) is a solution of the system (5.1)-(5.2) with the boundary and initial conditions (5.5)-(5.8) and satisfies the variational problem (corresponding to $(5.1)-(5.2)$ and $(5.5)-(5.8))$

$-\int_{Q_{T}} b\left(u_{\varepsilon}\right) \phi_{t} \mathrm{~d} x \mathrm{~d} t+\int_{Q_{T}}\left(a\left(\theta_{\varepsilon}^{\varepsilon}\right) \nabla u_{\varepsilon}+\boldsymbol{e}\left(\theta_{\varepsilon}^{\varepsilon}, u_{\varepsilon}\right)\right) \cdot \nabla \phi \mathrm{d} x \mathrm{~d} t=\int_{\Omega} b\left(u_{0}\right) \phi(0) \mathrm{d} x$

and

$$
\begin{aligned}
& -\int_{Q_{T}}\left(b\left(u_{\varepsilon}\right) \theta_{\varepsilon}+C_{S} \varrho_{S} \theta_{\varepsilon}\right) \psi_{t} \mathrm{~d} x \mathrm{~d} t+\int_{Q_{T}} \lambda\left(\theta_{\varepsilon}^{\varepsilon}, u_{\varepsilon}^{\varepsilon}\right) \nabla \theta_{\varepsilon} \cdot \nabla \psi \mathrm{d} x \mathrm{~d} t \\
& +\int_{Q_{T}}\left(\theta\left(a\left(\theta_{\varepsilon}^{\varepsilon}\right) \nabla u_{\varepsilon}+\boldsymbol{e}\left(\theta_{\varepsilon}^{\varepsilon}, u_{\varepsilon}\right)\right)\right) \cdot \nabla \psi \mathrm{d} x \mathrm{~d} t \\
& \quad=\int_{\Omega}\left(b\left(u_{0}\right) \theta_{0}+\rho \theta_{0}\right) \psi(0) \mathrm{d} x
\end{aligned}
$$

for all test functions $[\phi, \psi] \in C^{\infty}\left(\bar{Q}_{T}\right)^{2}, \phi=\psi=0$ in $\Gamma_{T}$ and $\phi(T)=\psi(T)=0$ in $\Omega$.

The above established convergences (6.11)-(6.14) and (6.19)-(6.22) are sufficient for taking the limit $\varepsilon_{j} \rightarrow 0$ as $j \rightarrow \infty$ in (6.23) and (6.24) (along a selected subsequence) to get the weak solution of the system (1.2)-(1.7) satisfying (4.1) and (4.2). This completes the proof of the main result stated by Theorem 4.2 .

\section{Applications}

Several engineering models of coupled groundwater flow and heat transport possess a common structure, derived from two balance equations of mass and heat energy $[9,23]$

$$
\partial_{t} \Theta(h)=\nabla \cdot(K(\theta, h) \nabla h)+\partial_{z} K(\theta, h)
$$

and

$$
\begin{aligned}
\partial_{t}\left(C_{w} \varrho_{w} \Theta(h) \theta+C_{S} \varrho_{S} \theta\right)= & \nabla \cdot(\Lambda(\theta, h) \nabla \theta) \\
& +\nabla \cdot\left(\theta C_{w} \varrho_{w}\left(K(\theta, h) \nabla h+e_{z} K(\theta, h)\right)\right) .
\end{aligned}
$$


Here the primary unknowns in the model are the absolute temperature $\theta=\theta(x, t)$ and the matric potential $h=h(x, t)[\mathrm{m}]$ (single-valued functions of the time $t$ and the spatial position $x \in \Omega) . K\left[\mathrm{~ms}^{-1}\right]$ represents the hydraulic conductivity of porous medium, $\lambda\left[\mathrm{W} \mathrm{m}^{-1} \mathrm{~K}^{-1}\right]$ is the effective thermal conductivity. Further, $\varrho_{w}\left[\mathrm{~kg} \mathrm{~m}^{-3}\right]$ is the density of liquid water, $C_{w}$ $\left[\mathrm{J} \mathrm{kg}^{-1} \mathrm{~K}^{-1}\right]$ represents the isobaric heat capacity of water, $\varrho_{S}\left[\mathrm{~kg} \mathrm{~m}^{-3}\right]$ and $C_{S}\left[\mathrm{~J} \mathrm{~kg}^{-1} \mathrm{~K}^{-1}\right]$, respectively, are the mass density and the isobaric heat capacity of solid microstructure. $\boldsymbol{e}_{z}$ stands for the vertical unit vector. The amount of water present at a certain matric potential of the porous medium is characterized by the water retention curve $\Theta=\Theta(h)[-]$. Here we present the relation proposed by van Genuchten and Mualem (see e.g. [11])

$$
\Theta(h)=\Theta_{r}+\left(\Theta_{s}-\Theta_{r}\right)\left[1+|\alpha h|^{n}\right]^{-m},
$$

where $\Theta_{s}$ is the soil saturated water content [-], $\Theta_{r}$ is the soil residual water content [-], $\alpha\left[\mathrm{m}^{-1}\right], m$ and $n$ are parameters.

The temperature dependence of hydraulic conductivity is given by $[9,23]$

$$
K(\theta, h)=K_{s} K_{r}(h) \frac{\nu\left(\theta_{0}\right)}{\nu(\theta)},
$$

$K_{s}\left[\mathrm{~m} \mathrm{~s}^{-1}\right]$ is the saturated hydraulic conductivity at the reference temperature $\theta_{0}, K_{r}\left[\mathrm{~m} \mathrm{~s}^{-1}\right]$ is the $h$-dependent relative hydraulic conductivity,

$$
K_{r}(h)=\sqrt{S(h)}\left(1-\left(1-S(h)^{1 / m}\right)^{m}\right)^{2}
$$

for $h<0$ (unsaturated porous media), $S(h)=\frac{\Theta(h)-\Theta_{r}}{\Theta_{s}-\Theta_{r}}$. Here $\Theta_{r}, \Theta_{s}, n$ and $m$ are positive constants. Finally, $\nu\left[\mathrm{m} \mathrm{s}^{-2}\right]$ is the temperature dependent kinematic viscosity.

Let us note that the equation (7.1) is degenerate with degeneracies in both elliptic and parabolic parts. It is a common treatment of nonlinear problems to make the so called Kirchhoff transformation, which employs the primitive function $\beta: \mathbb{R} \rightarrow \mathbb{R}^{+}, \zeta=\beta(\xi)$, defined by

$$
\beta(\xi)=\int_{0}^{\xi} K_{r}(s) \mathrm{d} s
$$

and converts these degeneracies only to the parabolic term (see [2]). This formally leads to the system (1.2)-(1.3), where

$$
\begin{aligned}
b(u) & :=\Theta\left(\beta^{-1}(u)\right), \\
a(\theta) & :=K_{s} \frac{\nu\left(\theta_{0}\right)}{\nu(\theta)}, \\
\rho & :=\frac{C_{S} \varrho_{S}}{C_{w} \varrho_{w}}, \\
\lambda(\theta, u) & :=\frac{\Lambda\left(\theta, \beta^{-1}(u)\right)}{C_{w} \varrho_{w}}, \\
e(\theta, u) & :=\frac{\boldsymbol{e}_{z} K\left(\theta, \beta^{-1}(u)\right)}{C_{w} \varrho_{w}} .
\end{aligned}
$$


Let us mention that the structure conditions (A1)-(A3) closely reflect the physical constraints on the mentioned transport model and experimental observations. The required regularity and boundedness of coefficients $a, b, \lambda$ and $\boldsymbol{e}$ are therefore ensured.

\section{Appendix A. The existence of the unique solution $u \in W_{\mathbf{2}}^{\mathbf{2 , 1}}\left(Q_{T}\right)$ to the system (5.15)-(5.17)}

As claimed in the proof of Theorem 5.1, Step 1, here we prove the following result.

Theorem A.1. There exists the uniquely determined $u \in W_{2}^{2,1}\left(Q_{T}\right)$, such that

$$
\begin{aligned}
\partial_{t} b(u) & =\nabla \cdot\left(a\left(\tilde{\theta}^{\varepsilon}\right) \nabla u+e\left(\tilde{\theta}^{\varepsilon}, u\right)\right) \quad \text { in } Q_{T}, \\
u & =0 \quad \text { in } \Gamma_{T}, \\
u(0) & =u_{0}^{\varepsilon} \quad \text { in } \Omega .
\end{aligned}
$$

Before we proceed to prove Theorem A.1, we explore some auxiliary results.

Proposition A.2. [2, Theorem 1.7, Remark 1.10] There exists the so-called weak solution $u \in L^{2}\left(I, W_{0}^{1,2}(\Omega)\right)$ to the problem (A.1)-(A.3), such that $b(u) \in$ $L^{\infty}\left(I, L^{1}(\Omega)\right), \partial_{t} b(u) \in L^{2}\left(I, W^{1,2}(\Omega)^{*}\right)$,

$$
\int_{0}^{T}\left\langle\partial_{t} b(u), \phi\right\rangle \mathrm{d} t+\int_{Q_{T}}\left(a\left(\tilde{\theta}^{\varepsilon}\right) \nabla u+\boldsymbol{e}\left(\tilde{\theta}^{\varepsilon}, u\right)\right) \cdot \nabla \phi \mathrm{d} x \mathrm{~d} t=0
$$

for all $\phi \in L^{2}\left(I, W_{0}^{1,2}(\Omega)\right)$ and

$$
\int_{0}^{T}\left\langle\partial_{t} b(u), \phi\right\rangle \mathrm{d} t+\int_{Q_{T}}\left(b(u)-b\left(u_{0}^{\varepsilon}\right)\right) \cdot \partial_{t} \phi \mathrm{d} Q_{T}=0
$$

for every test function $\phi \in L^{2}\left(I, W_{0}^{1,2}(\Omega)\right) \cap W^{1,1}\left(I, L^{\infty}(\Omega)\right)$ with $\phi(T)=0$.

The uniqueness of the weak solution is now proved in the following lemma.

Lemma A.3. The weak solution $u \in L^{2}\left(I, W^{1,2}(\Omega)\right)$ to the system (A.1)-(A.3) is unique.

Proof. Assume that there are two solutions $u_{1}$ and $u_{2}$ to the system (A.1)(A.3). For small $\epsilon>0$ let

$$
\psi_{\epsilon}(z):=\min \left(1, \max \left(\frac{z}{\epsilon}, 0\right)\right)
$$

and set $\phi:=\psi_{\epsilon}\left(u_{1}-u_{2}\right)$ as test function in the equation (A.4) to obtain

$$
\begin{gathered}
\int_{\Omega} \partial_{t}\left(b\left(u_{1}\right)-b\left(u_{2}\right)\right) \psi_{\epsilon}\left(u_{1}-u_{2}\right) \mathrm{d} x+\int_{\Omega} a\left(\tilde{\theta}^{\varepsilon}\right)\left|\nabla\left(u_{1}-u_{2}\right)\right|^{2} \psi_{\epsilon}^{\prime}\left(u_{1}-u_{2}\right) \mathrm{d} x \\
-\int_{\Omega} \nabla \cdot\left(\boldsymbol{e}\left(\tilde{\theta}^{\varepsilon}, u_{1}\right)-\boldsymbol{e}\left(\tilde{\theta}^{\varepsilon}, u_{2}\right)\right) \psi_{\epsilon}\left(u_{1}-u_{2}\right) \mathrm{d} x=0 .
\end{gathered}
$$


Let us mention that the second integral on the left hand side is nonnegative, therefore

$$
\begin{aligned}
& \int_{\Omega} \partial_{t}\left(b\left(u_{1}\right)-b\left(u_{2}\right)\right) \psi_{\epsilon}\left(u_{1}-u_{2}\right) \mathrm{d} x \\
& \quad \leq \int_{\Omega} \nabla \cdot\left(\boldsymbol{e}\left(\tilde{\theta}^{\varepsilon}, u_{1}\right)-\boldsymbol{e}\left(\tilde{\theta}^{\varepsilon}, u_{2}\right)\right) \psi_{\epsilon}\left(u_{1}-u_{2}\right) \mathrm{d} x .
\end{aligned}
$$

Passing to the limit for $\epsilon \rightarrow 0$, we have $\psi_{\epsilon} \rightarrow H$, where $H$ stands for the Heaviside function, and we arrive at the inequality

$$
\begin{aligned}
& \int_{\Omega} \partial_{t}\left(b\left(u_{1}\right)-b\left(u_{2}\right)\right) H\left(u_{1}-u_{2}\right) \mathrm{d} x \\
& \quad \leq \int_{\Omega} \nabla \cdot\left(\boldsymbol{e}\left(\tilde{\theta}^{\varepsilon}, u_{1}\right)-\boldsymbol{e}\left(\tilde{\theta}^{\varepsilon}, u_{2}\right)\right) H\left(u_{1}-u_{2}\right) \mathrm{d} x .
\end{aligned}
$$

Let $(w)_{+}=\max \{0, w\}$ and $(w)_{-}=\max \{0,-w\}$. Integration by parts yields

$$
\begin{gathered}
\int_{\Omega} \nabla \cdot\left(\boldsymbol{e}\left(\tilde{\theta}^{\varepsilon}, u_{1}\right)-\boldsymbol{e}\left(\tilde{\theta}^{\varepsilon}, u_{2}\right)\right) H\left(u_{1}-u_{2}\right) \mathrm{d} x \\
=\int_{\Omega} \nabla \cdot\left(\boldsymbol{e}\left(\tilde{\theta}^{\varepsilon}, u_{1}\right)-\boldsymbol{e}\left(\tilde{\theta}^{\varepsilon}, u_{2}\right)\right)_{+} \mathrm{d} x \\
=\int_{\Gamma}\left(\boldsymbol{e}\left(\tilde{\theta}^{\varepsilon}, u_{1}\right)-\boldsymbol{e}\left(\tilde{\theta}^{\varepsilon}, u_{2}\right)\right)_{+} \cdot \boldsymbol{n} \mathrm{d} S .
\end{gathered}
$$

Since $u_{1}=u_{2}$ on $\Gamma$, the last integral vanishes and (A.7)-(A.8) yield

$$
\int_{\Omega} \partial_{t}\left(b\left(u_{1}\right)-b\left(u_{2}\right)\right)_{+} \mathrm{d} x \leq 0 .
$$

Let us interchange $u_{1}$ and $u_{2}$ to get

$$
\int_{\Omega} \partial_{t}\left(b\left(u_{1}\right)-b\left(u_{2}\right)\right)_{-} \mathrm{d} x \leq 0 .
$$

Now the equations (A.9)-(A.10) yield $b\left(u_{1}\right)=b\left(u_{2}\right)$ and therefore $u_{1}=u_{2}$. The proof is complete.

We now prove the $L^{\infty}$-estimate for the weak solution to the system (A.1)(A.3). The following lemma together with the linear theory for parabolic problems (see [17, Chapter III.]) provides the a priori estimate (5.18).

Lemma A.4. Let $u$ be the weak solution to (A.1)-(A.3), then $u \in L^{\infty}\left(Q_{T}\right)$ and

$$
\|u\|_{L^{\infty}\left(Q_{T}\right)} \leq\left\|u_{0}^{\varepsilon}\right\|_{L^{\infty}(\Omega)} .
$$

Proof. Set

$$
\phi:=\left[b(u)-b\left(u_{*}\right)\right]_{-}= \begin{cases}b(u)-b\left(u_{*}\right), & u<u_{*}, \\ 0, & u \geq u_{*}\end{cases}
$$


as a test function in (A.4) to get

$$
\begin{aligned}
& \frac{1}{2} \int_{\Omega}\left[b(u)-b\left(u_{*}\right)\right]_{-}^{2} \mathrm{~d} x+\int_{Q_{t}} a\left(\tilde{\theta}^{\varepsilon}\right) b^{\prime}(u)|\nabla u|^{2} \chi_{\left\{u<u_{*}\right\}} \mathrm{d} x \mathrm{~d} s \\
& \quad-\int_{Q_{t}} \nabla \cdot \boldsymbol{e}\left(\tilde{\theta}^{\varepsilon}, u\right)\left[b(u)-b\left(u_{*}\right)\right]_{-} \mathrm{d} x \mathrm{~d} s=0 .
\end{aligned}
$$

Hence

$$
\begin{aligned}
& \frac{1}{2} \int_{\Omega}\left[b(u)-b\left(u_{*}\right)\right]_{-}^{2} \mathrm{~d} x+\int_{Q_{t}} a\left(\tilde{\theta}^{\varepsilon}\right) b^{\prime}(u)|\nabla u|^{2} \chi_{\left\{u<u_{*}\right\}} \mathrm{d} x \mathrm{~d} s \\
& \quad \leq \xi \int_{Q_{t}}\left|\nabla \cdot e\left(\tilde{\theta}^{\varepsilon}, u\right)\right|^{2} \mathrm{~d} x \mathrm{~d} s+C(\xi) \int_{Q_{t}}\left[b(u)-b\left(u_{*}\right)\right]_{-}^{2} \mathrm{~d} x \mathrm{~d} s
\end{aligned}
$$

and choosing $\xi$ sufficiently small we can use the Gronwall's lemma to conclude

$$
\frac{1}{2} \int_{\Omega}\left[b(u)-b\left(u_{*}\right)\right]_{-}^{2} \mathrm{~d} x=0 \quad \text { for almost every } t \in I .
$$

Now we conclude that the set $\left\{x \in \Omega ; u(x, t)<u_{*}\right\}$ has a measure zero for almost every $t \in I$.

Now setting

$$
\phi=\left[b(u)-b\left(u^{*}\right)\right]_{+}= \begin{cases}b(u)-b\left(u^{*}\right), & u>u^{*}, \\ 0, & u \leq u^{*}\end{cases}
$$

we obtain using similar arguments

$$
\frac{1}{2} \int_{\Omega}\left[b(u)-b\left(u^{*}\right)\right]_{+}^{2} \mathrm{~d} x=0 \quad \text { for almost every } t \in I .
$$
$t \in I$.

Hence the set $\left\{x \in \Omega ; u(x, t)>u^{*}\right\}$ has a measure zero for almost every

We are now in a position to prove Theorem A.1.

Proof. Let $u$ be the uniquely determined bounded weak solution to (A.1)-(A.3), which is ensured by Proposition A.2, Lemma A.3 and Lemma A.4. Consider the problem

$$
\begin{aligned}
b^{\prime}(u) \partial_{t} v-\nabla \cdot\left(a\left(\tilde{\theta}^{\varepsilon}\right) \nabla v\right) & =\nabla \cdot \boldsymbol{e}\left(\tilde{\theta}^{\varepsilon}, u\right) \quad \text { in } Q_{T}, \\
v & =0 \quad \text { in } \Gamma_{T}, \\
v(0) & =u_{0}^{\varepsilon} \quad \text { in } \Omega,
\end{aligned}
$$

where $\nabla \cdot \boldsymbol{e}\left(\tilde{\theta}^{\varepsilon}, u\right) \in L^{2}(\Omega)$. Let us stress that there exists some positive constant $b_{0}$, such that

$$
0<b_{0} \leq b^{\prime}(u) \leq b_{2}<+\infty
$$

as a result of Lemma A.4 and the assumption (3.2). By [17, Chapter III., Theorem 6.1. and Remark 6.2] the system (A.14)-(A.16) possesses just one weak solution

$$
v \in W_{2}^{1,1}\left(Q_{T}\right)
$$


Now we write the system (A.14)-(A.16) in the form

$$
\begin{aligned}
\partial_{t} v-\Delta v & =f \quad \text { in } Q_{T}, \\
v & =0 \quad \text { in } \Gamma_{T}, \\
v(0) & =u_{0}^{\varepsilon} \quad \text { in } \Omega,
\end{aligned}
$$

where

$$
f:=\partial_{t} v-\frac{b^{\prime}(u)}{a\left(\tilde{\theta}^{\varepsilon}\right)} \partial_{t} v+\frac{1}{a\left(\tilde{\theta}^{\varepsilon}\right)} \nabla a\left(\tilde{\theta}^{\varepsilon}\right) \cdot \nabla v+\frac{1}{a\left(\tilde{\theta}^{\varepsilon}\right)} \nabla \cdot \boldsymbol{e}\left(\tilde{\theta}^{\varepsilon}, u\right) .
$$

By (A.17), (A.18) and the assumptions (A1)-(A2) we have $f \in L^{2}\left(Q_{T}\right)$. Following the linear theory for parabolic equations [17, Chapter III.] we get the uniquely determined strong solution of the system (A.19)-(A.21)

$$
v \in W_{2}^{2,1}\left(Q_{T}\right) \text {. }
$$

This yields the regularity $v \in W_{2}^{2,1}\left(Q_{T}\right)$ for the system (A.14)-(A.16). By the uniqueness of the solution $v$ to the system (A.14)-(A.16) and the uniqueness of the weak solution $u$ to the system (A.1)-(A.3) we conclude $u=v \in W_{2}^{2,1}\left(Q_{T}\right)$. This concludes the proof of Theorem A.1.

\section{Acknowledgements}

This research was supported by the project GAČR 13-18652S (author one) and by the grant SGS13/001/OHK1/1T/11 provided by the Grant Agency of the Czech Technical University in Prague (author two).

\section{References}

[1] Adams, A., Fournier, J.F.: Sobolev spaces. In: Pure and Applied Mathematics, vol. 140. Academic Press, New York (2003)

[2] Alt, H.W., Luckhaus, S.: Quasilinear elliptic-parabolic differential equations. Math. Z 183, 311-341 (1983)

[3] Aubin, J.P.: Un théorème de compacité. C. R. Acad. Sci. Paris 256, 50425044 (1963)

[4] Beneš, M.: A note on doubly nonlinear parabolic systems with unilateral constraint. Results Math. 63, 47-62 (2013)

[5] Beneš, M., Štefan, R.: Global weak solutions for coupled transport processes in concrete walls at high temperatures. ZAMM. Z. Angew. Math. Mech. 93, 233-251 (2013)

[6] Beneš, M., Zeman, J.: Some properties of strong solutions to nonlinear heat and moisture transport in multi-layer porous structures, Nonlin. Anal. RWA 13, 1562-1580 (2012) 
[7] Černý, R., Rovnaníková, P.: Transport Processes in Concrete. CRC Press, Boca Raton (2002)

[8] Dalík, J., Daněček, J., Vala, J.: Numerical Solution of the Kiessl Model. Appl. Math. 45, 3-17 (2000)

[9] Deangelis, M.L., Wood, E.F.: A detailed model to simulate heat and moisture transport in a frozen soil. Hydrology, Water Resources and Ecology in Headwaters, IAHS Publ. no. 248 (1998)

[10] Filo, J., Kačur, J.: Local existence of general nonlinear parabolic systems. Nonlinear Anal. 24, 1597-1618 (1995)

[11] van Genuchten, M.T.: A closed form equation for predicting the hydraulic conductivity of unsaturated soil. Soil Sci. Soc. Am. J. 44, 892-898 (1980)

[12] Giaquinta, M., Modica, G.: Local existence for quasilinear parabolic systems under nonlinear boundary conditions. Ann. Mat. Pura Appl. 149, 41-59 (1987)

[13] Gilbarg, D., Trudinger, N.S.: Elliptic partial differential equations of second order. Springer, Berlin (2001)

[14] Hömberg, D.: A mathematical model for the phase transitions in eutectoid carbon steel. IMA J. Appl. Math. 54, 31-57 (1995)

[15] Kačur, J.: On a solution of degenerate elliptic-parabolic systems in OrliczSobolev spaces. I. Math. Z 203, 153-171 (1990)

[16] Kufner, A., John, O., Fučík, S.: Function Spaces. Academia, New York

[17] Ladyzhenskaya, O.A., Solonnikov, V.A., Uraltseva, N.N.: Linear and quasilinear equations of parabolic type. In: Translations of Mathematical Monographs, vol. 23. American Mathematical Society, New York

[18] Li, B., Sun, W.: Global existence of weak solution for nonisothermal multicomponent flow in porous textile media. SIAM J. Math. Anal. 42, 3076-3102 (2010)

[19] Li, B., Sun, W., Wang, Y.: Global existence of weak solution to the heat and moisture transport system in fibrous porous media. J. Differ. Equ. 249, 26182642 (2010)

[20] Li, B., Sun, W.: Global weak solution for a heat and sweat transport system in three-dimensional fibrous porous media with condensation/evaporation and absorption. SIAM J. Math. Anal. 44, 1448-1473 (2012)

[21] Nečas, J.: Introduction to the theory of nonlinear elliptic equations. TeubnerTexte zur Mathematik, Leipzig (1983)

[22] Merz, W., Rybka, P.: Strong solution to the Richards equation in the unsaturated zone. J. Math. Anal. Appl. 371, 741-749 (2010)

[23] Scanlon, B.R., Milly, P.C.D.: Water and heat fluxes in desert soils: 2. Numerical simulations. Water Resour. Res. 30, 721-733 (1994) 
[24] Lewis, R.W., Schrefler, B.A.: The finite element method in the static and dynamic deformation and consolidation of porous media, John Wiley and Sons, Ltd. Baffins Lane, Chichester (1998)

[25] Vala, J.: On a system of equations of evolution with a non-symmetrical parabolic part occuring in the analysis of moisture and heat transfer in porous media. Appl. Mat. 47, 187-214 (2002)

[26] Weidemaier, P.: Local existence for parabolic problems with fully nonlinear boundary condition: an $L_{p}$-approach. Ann. Mat. Pura Appl. 160, 207-222 (1991)

Michal Beneš and Lukáš Krupička

Department of Mathematics

Faculty of Civil Engineering

Czech Technical University in Prague

Thákurova 7

16629 Prague 6

Czech Republic

e-mail: benes@mat.fsv.cvut.cz

Lukáš Krupička

e-mail: luk.krupicka@gmail.com

Received: 4 February 2014.

Accepted: 3 June 2014. 